\title{
UNA APROXIMACIÓN A UN MODELO DE CONOCIMIENTO DEL FORMADOR DE PROFESORES DE MATEMÁTICAS
}

\author{
AN APPROACH TO A MODEL OF MATHEMATICS \\ TEACHER EDUCATOR KNOWLEDGE
}

\section{UMA APROXIMAÇÃO A UM MODELO DE CONHECIMENTO DO EDUCADOR DE PROFESSORES DE MATEMÁTICA}

\author{
Luis Carlos Contreras \\ Universidad de Huelva, Huelva, España \\ Recibido: 01/03/2021 - Aceptado: 18/04/2021 - Publicado: 03/05/2021 \\ Remita cualquier duda sobre esta obra a: Luis Carlos Contreras. \\ Email:Lcarlos@ubu.es
}

\begin{abstract}
RESUMEN
Este artículo es una revisión teórica acerca del conocimiento del formador de profesores de matemáticas. Décadas de estudio acerca del conocimiento del profesor han producido abundante información teórica y empírica acerca del mismo y algunos grupos que han contribuido a su desarrollo se plantean ahora avanzar hacia modelos de conocimiento de los formadores, convirtiendo este ámbito en prioritario. Se tiende a elaborar estos modelos desde la perspectiva teórica subyacente de un determinado modelo de conocimiento del profesor, admitiendo que ambos conocimientos (el del formador y el del profesor) son sustancialmente diferentes, aunque de intersección no vacía. En este artículo, el modelo de conocimiento del profesor que inspira el trabajo es el del conocimien to especializado del profesor de matemáticas (MTSK); de ahí que este sea el punto de partida de la reflexión que se presenta. El propio contenido de la formación y la consideración de los diferentes perfiles de quienes han de desarrollarla servirán de base para la propuesta de dominios del conocimiento que se presenta.
\end{abstract}

Palabras clave: Conocimiento del formador; Conocimiento del profesor de matemáticas; MTSK, MTEK

\begin{abstract}
This article is a theoretical review about the mathematics teacher educator knowledge. Decades of study on teacher knowledge have produced abundant theoretical and empirical information about it and some groups that have contributed to its development are now considering moving towards teacher educator knowledge models, making this area a priority. These models tend to be elaborated from the underlying theoretical perspective of a certain model of teacher knowledge, admitting that both knowledge (that of the teacher educator and that of the teacher) are substantially different, although they have common elements. In this article, the model of teacher
\end{abstract}


knowledge that inspires the work is that of the mathematics teacher's specialized knowledge (MTSK); hence this is the starting point of the reflection that is presented. The content of the training itself and the consideration of the different profiles of those who have to develop it will serve as the basis for the proposed domains of knowledge that are presented.

Keywords: Educator knowledge; Mathematics teacher' knowledge; MTSK, MTEK.

\section{RESUMO}

Este artigo é uma revisão teórica sobre os saberes do formador de professores de matemática. Décadas de estudo sobre o conhecimento do professor produziram abundantes informaçóes teóricas e empíricas sobre o mesmo e alguns grupos que contribuíram para o seu desenvolvimento estão agora considerando avançar para modelos de conhecimento do formador, tornando esta área uma prioridade. Esses modelos tendem a ser elaborados a partir da perspectiva teórica subjacente a um determinado modelo de conhecimento do professor, admitindo que ambos os saberes (do formador e do professor) são substancialmente diferentes, embora tenham elementos comuns. Neste artigo, o modelo de conhecimento do professor que inspira o trabalho é o do saber especializado do professor de matemática (MTSK); portanto, este é o ponto de partida da reflexão que se apresenta. O conteúdo da própria formação e a consideração dos diferentes perfis de quem deve desenvolvê-la servirão de base aos domínios de conhecimento propostos que se apresentam.

Palavras-chave: Conhecimento do formador; Conhecimento do professor de matemática; MTSK, MTEK.

\section{INTRODUCCIÓN}

La investigación en Educación Matemática ha dedicado grandes esfuerzos, en las últimas cuatro décadas a comprender el conocimiento del profesor, en general, y del profesor de matemáticas en particular. Hablamos de profesor de matemáticas en dos contextos que tienen regulación diferente. La del maestro de Primaria, que en la mayoría de los países tiene una formación generalista (podíamos hablar de él como el profesor que enseña matemáticas, entre otras materias curriculares), y la del profesor de Secundaria o Bachillerato, que enseña fundamentalmente matemáticas, con una formación en matemáticas, ciencias o ingeniería y una especialización (de contenido variable, según países) en educación matemática. Observemos que, incluso en estos casos, no existe un modelo generalizado que ampare el conocimiento del profesor; conviven diversos modelos (Ball et al., 2008; Godino, 2009; Carrillo et al., 2018) nacidos con fines analíticos para dar respuesta a la pregunta ¿qué debe conocer un profesor que ha de enseñar matemáticas?, modelos con importantes puntos de convergencia que podrían servir de fundamento para unos estándares curriculares compartidos en la formación de profesores de matemáticas.

El caso del formador es todavía más complejo. El abanico de posibilidades para definir quién es un formador de profesores de matemáticas (en adelante MTE) es mucho más amplio y, por tanto, caracterizar su conocimiento bastante más complejo. El objetivo de este artículo es aportar elementos que ayuden a reflexionar sobre ello. 
El interés de la investigación en el conocimiento del formador de profesores de matemáticas (en adelante MTEK) es creciente. Como señalan Chick y Beswick (2018), se ha puesto en duda la suposición predominante de que haber sido un excelente profesor de matemáticas capacita para formar profesores de matemáticas, argumentando que el conocimiento de un profesor de matemáticas, en particular en lo concerniente al conocimiento didáctico del contenido, no es suficiente para el formador de profesores. Asumimos que la competencia como profesor de matemáticas escolares es condición necesaria, pero no suficiente, de la misma forma que la competencia en matemáticas es condición necesaria, pero no suficiente, para los profesores de matemáticas.

Algunas investigaciones (Zaslavsky \& Leikin, 2004) han puesto de manifiesto que el conocimiento de los MTE podría ser una extensión del conocimiento de los profesores de matemáticas, abarcando ese conocimiento, de la misma manera que el conocimiento de los profesores de matemáticas ha de contender el conocimiento que pretenden construir en sus estudiantes. En ese mismo sentido, Castro-Superfine et al. (2020) intentan mostrar el conocimiento del profesor como uno de los subdominios del conocimiento del formador (knowledge of mathematical knowledge for teaching). Compartiendo la idea de que parte del contenido de la formación de profesores es el conocimiento didáctico del contenido matemático ( $\mathrm{PCK}$, donde $\mathrm{C}$ es el contenido matemático), el conocimiento para su enseñanza será una especie de meta-PCK que podría describirse como P”C”K (donde “C” seria el contenido de la formación) para enseñar cada uno de los subdominios del conocimiento del profesor (Chick \& Beswick, 2013, lo denominan MTEPCK).

Esta perspectiva invita a pensar en un modelo del conocimiento del formador inspirado, de alguna manera, en un modelo de conocimiento del profesor. Aunque en este artículo, nuestro modelo de conocimiento del profesor, MTSK (Carrillo et al., 2018), que va a estar presente, y ocupará el siguiente apartado con objeto de mejorar la contextualización de nuestra propuesta, nuestra intención es avanzar hacia un modelo de conocimiento del formador sin que ello sea una restricción. Así, tras mostrar el modelo MTSK, comenzaremos nuestra reflexión poniendo la mirada en las características de aquellos que denominaremos formadores, describiendo su tipología y sus perfiles formativos, entraremos después en una caracterización del contenido de la formación, basada en estándares internacionales, para, de ahí pasar a describir algunos subdominios de un modelo de conocimiento del formador que permitirían desarrollar esos estándares.

\section{EL MODELO DE CONOCIMIENTO ESPECIALIZADO DEL PROFESOR DE MATEMÁTICAS (MTSK)}

En 1986, Lee Shulman, comenzaba su conocido artículo en la American Educational Research Association con un demoledor aforismo de Bernard Shaw: "El que puede, hace; el que no, enseña". Mostraba así su forma de ver el valor social que se concedía (se podría decir, en presente, se concede) a 
los profesores. Desgranaba luego las razones, analizando las reformas curriculares de los 70 ' Y los 80 ' en los Estados Unidos y ponía de relieve una gran brecha entre el conocimiento disciplinar y el conocimiento de pedagogía, desarrollado cada uno a espaldas del otro y que los sistemas educativos han pretendido hacer converger en forma de mezcla o cóctel. Se preguntaba, más adelante, si esta tendencia era reciente o venía del pasado, y exponía cómo en las universidades medievales se preparaba "ad hoc" para el más alto grado universitario, La Maestría, donde el conocimiento disciplinar y la enseñanza se fusionaban al estilo de la más pura tradición aristotélica, poniendo de relieve que la mejor forma de evaluar el conocimiento que alguien tiene es cuando vemos cómo lo enseña. Se preguntaba entonces cómo volver a prestigiar al profesor, cómo volver a construir ese tipo de conocimiento; en definitiva, cómo hacer que un experto en el contenido se transforme en un experto en hacerlo comprender.

Al final de su trabajo retoma la frase de Bernard Shaw y la transforma, apuntando hacia "el paradigma perdido": "El que puede, hace; el que comprende, enseña” (Shulman, 1986; p. 14), con una reflexión previa en la que, recordando de nuevo a Aristóteles, afirma que la comprensión no es otra cosa que la capacidad de transformar el saber disciplinar en un conocimiento enseñable.

Esta capacidad de transformar el conocimiento de la disciplina es lo que hoy denominamos Conocimiento Didáctico del Contenido. No se trata de una intersección entre el conocimiento pedagógico general (pedagogical knowledge) y el conocimiento disciplinar (subject mattet knowledge), sino más bien parte del propio conocimiento de la disciplina, considerado como objeto de enseñanza y aprendizaje (subject matter knowledge for teeabing). Se trata de un conocimiento que "incluye las más útiles formas de representación, las más poderosas analogías, ilustraciones y ejemplos, explicaciones y demostraciones, en una palabra, las formas de representar y reformular el conocimiento para hacerlo comprensible a otros” (p. 9). naturalmente, este planteamiento implica que más que hablar de conocimiento del profesor (con carácter general) es preciso hablar de conocimiento del profesor relativo a una disciplina específica, poniendo de relieve la dependencia directa de la misma y, por tanto, la no adaptabilidad automática de una a otra.

Pero no es esta la única aportación de Shulman al conocimiento del profesor. También nos hace ver que el propio conocimiento del contenido es preciso contemplarlo desde su organización y estructura en la mente del profesor y, citando a Schwab (1978), establece dos grandes subdominios de este conocimiento, el sustantivo y el sintáctico:

El profesor no solo necesita entender que algo es asi; debe comprender mejor por quées asi, por qué motivos se puede afirmar su orden y en quécircunstancias nuestra creencia en su justificación puede debilitarse e incluso negarse. Además, esperamos que el profesor comprenda por qué un tema dado es particularmente central para una disciplina, mientras que otro puede ser periférico. Esto será importante en juicios pedagógicos posteriores con respecto al énfasis curricular relativo. (Shulman, 1986, p. 9) 
Como aclara un año después (Shulman, 2005), de todos los conocimientos del profesor, "El conocimiento didáctico del contenido es la categoría que, con mayor probabilidad, permite distinguir entre la comprensión del especialista en un área del saber y la comprensión del didacta” (p. 11).

Es probable que esta frase, en la que se establecen diferentes formas de conocer la disciplina, detonara en el grupo de Deborah Ball, en la Universidad de Michigan, los estudios que condujeron al Mathematical Kowledge for Teaching (MKT). A este grupo, que se apoya en los hombros de Shulman, desde la perspectiva del conocimiento matemático, debemos varias aportaciones relevantes. La primera, ya esbozada, es la distinción entre conocimiento matemático común (a cualquier persona matemáticamente instruida) y conocimiento matemático especializado (específico del profesor); la segunda, la idea de conocimiento en el horizonte matemático (HCK).

En Ball y Bass (2009) se establecían tres subdominios dentro del HCK: HCK (T), HCK (P) y HCK (V). El HCK(T) incluye el conocimiento de las principales ideas y estructuras de la disciplina y las conexiones entre diferentes entes matemáticos, y el desarrollo de nuevos entes a partir del conocimiento existente. Incluye también el conocimiento de conexiones con contenidos posteriores y anteriores a los que se están tratando. En el HCK(P) está el conocimiento de las formas de conocer y crear o producir en Matemáticas, aspectos de la comunicación matemática, el razonamiento y la prueba, saber definir y usar definiciones, establecer relaciones (entre conceptos, propiedades, etc.), correspondencias o equivalencias o elegir representaciones, generalizar o explorar. En el $\mathrm{HCK}(\mathrm{V})^{1}$ se consideran los valores centrales de la disciplina, como la precisión y el cuidado con la consistencia del lenguaje matemático, el gusto por la coherencia argumental, la corrección y la exactitud como opuesto de la ambigüedad (no como opuesto de la aproximación). La idea de matemática elemental desde un punto de vista avanzado y matemática avanzada desde un punto de vista elemental parece ser el elemento de conexión más potente entre estos tres subdominios.

Como decíamos en Carrillo et al. (2013), estos tres subdominios del HCK tienen, desde nuestra perspectiva, una naturaleza sustancialmente diferente y, además, abarcan todos los aspectos del conocimiento matemático (MK). HCK(T) tiene, a su vez, dos núcleos claramente diferenciados, los elementos principales de la disciplina y la estructura de la misma, por lo que estos dos núcleos tienen capacidad de contener todo el conocimiento matemático común desde la perspectiva del quées la matemática, vista como un conjunto de Temas incardinados en una Estructura, mientras el HCK (P) contendría los conocimientos relativos al cómo se construye. En cuanto a la diferenciación entre conocimiento común o especializado, nos lleva a reflexionar acerca de carácter especializado de todo el modelo en el sentido de que conocimiento profesional es aquél que está al servicio del trabajo diario del profesor.

\footnotetext{
${ }^{1}$ Vinculamos el HCK (V) a las concepciones y creencias. De todas formas, como la precisión, la consistencia del lenguaje y el gusto por la coherencia argumental son comunes a todo el pensamiento científico y no exclusivas de la educación matemática, no aparecerá de forma explícita en el modelo.
} 
Hay otros dos subdominios en el modelo de Michigan que merecen nuestra atención, son el conocimiento de las matemáticas y la enseñanza y el conocimiento de las matemáticas y los estudiantes. Resulta atrayente que, en ambos casos, la matemática es vista como objeto de enseñanza y de aprendizaje (cuando se piensa en los estudiantes). Está claro que el profesor necesita conocer las dificultades más frecuentes con las que se enfrentan los estudiantes a un contenido. Por ejemplo, debe conocer que es probable que piensen que si dos figuras planas tienen la misma superficie (como es el caso de B y D, en la Figura 1), entonces también tienen el mismo perímetro (o viceversa) o que, derivado de esto, piensen que, dado que algunas de las áreas de estas figuras son diferentes ( $\mathrm{A}$ y $\mathrm{C}$, entre sí, y ambas diferentes de B y D), sus perímetros han de ser también diferentes (ver Figura 1).

\section{Figura 1:}

Un problema con el que evidenciar las incorrectas relaciones área-perimetro en figuras planas. (INECSE, 2005)

Un carpintero tiene 32 metros de madera y quiere construir una pequeña valla alrededor de un parterre en el jardín. Está considerando los siguientes diseños para el parterre.
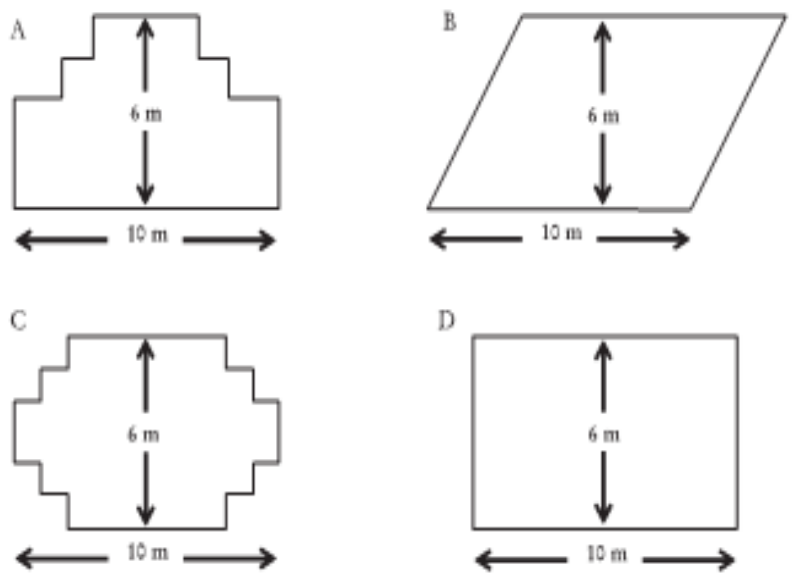

Asimismo, debe saber que dispone de múltiples recursos para abordar esta cuestión y de cada uno de ellos ha de conocer sus posibilidades y limitaciones. Que, por ejemplo, con las Geotiras puede construir un paralelogramo y, moviendo la articulación construida, puede modificar los valores de los ángulos interiores del mismo, mostrando con ello que un mismo perímetro puede encerrar diferentes áreas. Del mismo modo, debe saber que la relación contraria (que a igual área encerrada no tiene por qué corresponder un mismo perímetro) no se evidencia fácilmente con este recurso, debiendo recurrir a otros como Tangram o Poliminós.

A pesar de habernos servido de inspiración, la diferenciación entre conocimiento matemático común y especializado, presenta serias dificultades en el ámbito analítico. La mera explicación de que común es aquél conocimiento matemático que posee cualquier persona matemática instruida no nos permite diferenciar con precisión, cuando analizamos la acción del profesor en el aula, y lo hace depender 
de muchos factores extrínsecos (nivel educativo, sistema educativo de cada país, contexto sociocultural...). Además, en determinadas situaciones, el conocimiento matemático especializado se convertía en una especie de "cajón de sastre" en el que se ubicaban conocimientos específicos del profesor de matemáticas que tenían difícil encaje en otros subdominios del modelo de Michigan. Por ejemplo, en Ball y Bass (2002), se pone como ejemplo de conocimiento especializado (y se subraya su carácter matemático, y no pedagógico), el que permite al profesor conocer como alternativos los algoritmos B y C de la Figura 2.

\section{Figura 2}

Algoritmos alternativos del producto (Ball E Bass, 2002)

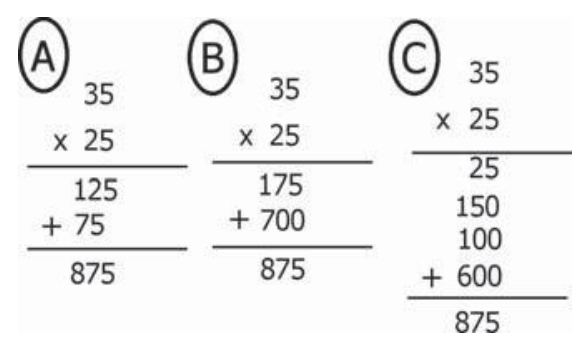

Coincidimos con el carácter matemático (y no pedagógico) de este conocimiento; sin embargo, no compartimos que sea exclusivo del profesor. Quizás el hecho de que otras personas no lo conozcan puede deberse al tratamiento mecánico del cálculo aritmético escolar en la mayor parte de los sistemas educativos del mundo, a la identificación de la operación con el algoritmo, al uso de algoritmos sin desvelar y comprender sus pasos ocultos. Es cierto que a un ciudadano formado le basta con conocer uno y que no necesita más para desenvolverse en la vida, pero no nos parecen suficientes razones para, por ello, considerar que entonces es específico solo de aquél que lo usa en su trabajo. Además, si analizamos la segunda parte de la justificación de este ítem de conocimiento hecha por sus autores, incluye argumentos que permitirían incluirlo en conocimiento de las matemáticas y su enseñanza y en conocimiento de las matemáticas y los estudiantes (dos subdominios de su modelo MKT):

Un profesor debe poder preguntar qué sucede en cada uno de estos enfoques y saber cuál de ellos es un método que funciona para multiplicar dos números enteros. Estas son preguntas matemáticas por excelencia, no pedagógicas. Saber preguntar y cómo responder a tales preguntas matemáticas es esencial para poder hacer juicios sabios en la enseñanza. Por ejemplo, una decisión sobre si examinar o no estos métodos alternativos con los estudiantes depende de primero evaluar los problemas matemáticos involucrados en el enfoque particular, y si ofrecen posibilidades de aprendizaje matemático valioso para estos estudiantes en este momento. (p. 7) 
Desde nuestro punto de vista es más operativo considerar como especializado a todo el conocimiento de un profesor de matemáticas. Así, en relación con el procedimiento señalado, el profesor debe conocer no sólo cómo se hace, sino también cuándo puede hacerse, por quése bace de esas formas y cómo evaluar el resultado. Todo ello formaría parte de la categoría Procedimientos del subdominio Conocimiento de los Temas, donde también incluimos la Fenomenología y las aplicaciones de los conceptos, los diferentes registros de representación y las definiciones, las propiedades y sus fundamentos. Junto con el conocimiento de la Estructura de la Matemática y el conocimiento de la Práctica Matemática (ya comentados anteriormente, como frutos del HCK) conforman todo el Dominio Matemático.

Aunque puede parecer una modificación puramente terminológica respecto de MKT, preferimos hablar de conocimiento de las Caracteristicas del aprendizaje de las Matemáticas (que incluye conocimiento sobre las teorias de aprendizaje, formales o personales, el conocimiento de las fortalezas y dificultades que suelen tener los estudiantes ante un determinado contenido, su forma de interaccionar con el mismo y los intereses y expectativas con que se enfrentan a su aprendizaje) en vez de un conocimiento intersección entre las matemáticas y los estudiantes, así como conocimiento de la Enseñanza de las Matemáticas (que incluye el conocimiento de las teorias de enseñanza, formales o personales, el conocimiento de los recursos y de las estrategias, técnicas, tareas y ejemplos) en vez de un conocimiento intersección de las matemáticas y la enseñanza, unificando ambos subdominios en una visión de la matemática como objeto de enseñanza y aprendizaje (ver Figura 3). Finalmente, en el centro del modelo, situamos las creencias del profesor sobre la matemática y sobre su enseñanza que permean todo el conocimiento en su conjunto.

\section{Figura 3}

El modelo MTSK (Carrillo et al., 2018)

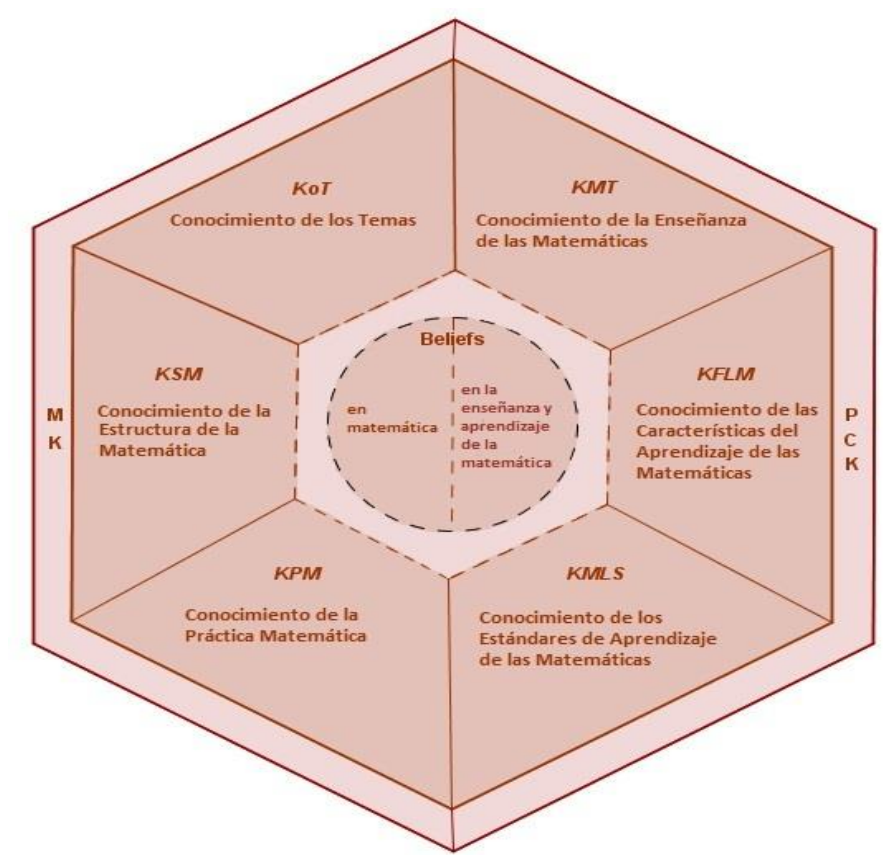


El modelo disecciona artificialmente el conocimiento para su mejor comprensión, pero a la vez lo entiende integrado y relacionado. Una forma de ver estas relaciones es la categoría estrategias, técnicas, tareas y ejemplos. El conocimiento de un espacio de ejemplos en relación con un determinado concepto forma parte del Conocimiento de los Temas, pero cuando un profesor selecciona de ese espacio aquellos ejemplos que considera adecuados para un determinado momento, y los secuencia en el proceso de enseñanza, moviliza conocimiento sobre la Enseñanza de las Matemáticas; si preguntamos al profesor las razones de una secuencia y no otra, será fácil encontrar argumentos relativos a las dificultades de aprendizaje de los estudiantes (Conocimiento de las Características del aprendizaje de las Matemáticas) y si usa contraejemplos para refutar una afirmación de un estudiante, evidencia el conocimiento de una forma de validación, propia del conocimiento de la Práctica Matemática. En una misma actuación docente, el profesor evidencia conocimientos vinculados que pertenecen a diferentes subdominios de MTSK.

MTSK aporta una nueva organización del conocimiento del profesor de matemáticas y, principalmente, aporta su consideración global como especializado. La organización está realizada desde una perspectiva intrínseca del conocimiento matemático, visto como objeto de enseñanza y aprendizaje, que nos permite una mejor caracterización de los subdominios; incluye además las creencias, como un elemento del pensamiento del profesor que condiciona la organización de su conocimiento. Naturalmente está elaborado de nuestro paradigma de investigación y desde nuestras propias creencias cerca de la matemática y de sus procesos de enseñanza y aprendizaje.

Hemos usado MTSK para analizar y comprender el conocimiento de profesores de matemáticas de Educación Infantil, Educación Primaria, Educación Secundaria y Universidad, en diferentes contextos educativos y cuando enseñaban diferentes contenidos. Ello nos ha permitido afianzarnos en la validez del modelo a la vez que hemos descifrado elementos del conocimiento del profesor para la gestión de determinados contenidos matemáticos en su aula. Estamos ahora profundizando en ese aspecto, analizando el MTSK que se moviliza en la gestión de problemas clásicos de enseñanza y aprendizaje, como la definición y clasificación de polígonos, la relación área-perímetro en las figuras planas, las operaciones con fracciones o la resolución de problemas. Hemos comenzado a diseñar actividades de formación de profesores en todos los niveles y el objetivo en el medio plazo es que los logros alcanzados nos permitan diseñar programas completos de formación de profesores de matemáticas basados en la estructura y filosofía del modelo. Ahora, como expondremos a continuación, hemos comenzado a reflexionar sobre la naturaleza y estructura del conocimiento del formador de profesores de matemáticas que tendrían que desarrollar esos programas. Abordaremos esta cuestión desde dos perspectivas; primero intentaremos caracterizar a los actores que ejecutan la formación, dado que sus perfiles profesionales condicionarán sus conocimientos; luego analizaremos el contenido de la formación que deberían desarrollar estos formadores para, sobre estos dos pilares, avanzar en la construcción de los conocimientos del formador que serían necesarios para ello. 


\section{¿QUIÉN ES UN FORMADOR?}

La respuesta a esta pregunta es compleja. Dependiendo del contexto (ni siquiera del país) son, de facto, formadores de profesores de matemáticas: matemáticos (no especialistas en Educación Matemática, cuyo perfil es el de un investigador en matemáticas); matemáticos o graduados en educación (cuyo perfil es el de un investigador en educación Matemática), matemáticos o graduados en ciencias o ingeniería, que ejercen como profesores de matemáticas; graduados en educación (con un perfil investigador de Ciencias de la Educación), entre otros. Es posible que esto explique las nomenclaturas que se han barajado en las aproximaciones al problema que nos planteamos: Mathematics Teacher Educator Knowledge (Jaworski, 2008), Mathematical Knowledge for Teaching Teachers (Zopf, 2010), Knowledge of Mathematics Teacher Trainer, o Mathematics' Teacher Trainer Specialized Knowledge.

Appova y Taylor (2019) optan por la definición de Jaworski (2008), entendiendo formador como el "profesional que trabaja con profesores en ejercicio o en formación para desarrollar y mejorar la enseñanza de las matemáticas” (Jaworski, 2008, p. 1), que constituye una visión inclusiva de aquellos que trabajamos en la formación de profesores de matemáticas.

Decía Bass (1997), quien fue presidente del ICMI, que muchos matemáticos “profesionales” (que el denomina científicos matemáticos) han tendido a mirar a los educadores matemáticos con dudas rayanas en el desprecio; él, sin embargo, confiesa que todos tenemos mucho que aprender de los demás y que todavía pueden mejorarse los contextos para una comunicación respetuosa y colaboración profesional entre científicos matemáticos y educadores matemáticos, desde maestros de Educación Primaria, hasta personas que hacen investigación en educación. Todos caminamos en un largo camino en el que los científicos matemáticos pueden contribuir al fortalecimiento de programas escolares y práctica docente, desde el punto de vista de la disciplina, mientras que las comunidades de investigación en educación matemática, pueden elevar la conciencia pedagógica y competencia de los científicos matemáticos académicos. Desde esta perspectiva podría decirse que todos podrían contribuir en la formación de profesores de matemáticas, aunque la conciencia de una labor profesional específica y el consecuente reconocimiento de un conocimiento matemático visto como objeto de enseñanza aprendizaje se revela primordial. Sin duda esa visión puede venir muy sesgada dependiendo de la formación inicial y la labor profesional desarrollada, dado que estos dos aspectos pueden condicionar las propias concepciones y la identidad profesional.

Aunque en el párrafo anterior parecen recogerse exclusivamente a los formadores que trabajan desde la "academia" (profesores universitarios), no queremos olvidar a otros profesionales que colaboran en la formación práctica, como tutores o mentores. Todos, y cada uno de ellos, pueden y tienen algo que aportar en la formación de profesores de matemáticas, solo han de preguntarse qué pueden y deben aportar en la construcción de un conocimiento de carácter profesional. 
La investigación empírica deberá mostrarnos la incidencia de los perfiles que hemos señalado y la contribución esperable de cada uno de ellos en el desarrollo de programas formativos. En el apartado siguiente nos ocuparemos del contenido que deberían tener esos programas.

\section{¿QUÉ CARACTERÍSTICAS HA DE TENER EL CONTENIDO DE LA FORMACIÓN?}

Ante la existencia de modelos de análisis del conocimiento del profesor de matemáticas, que se han ido utilizando como organizadores del diseño de programas formativos, parece razonable apoyarse en ellos para dar respuesta a nuestra pregunta. Puede adoptarse una postura ecléctica, tomando de cada uno de ellos los elementos más acordes con los paradigmas que uno comparte o, por el contrario, se puede abrazar de forma específica uno de ellos, sobre todo, como es nuestro caso, cuando se ha contribuido al desarrollo de un modelo, en este caso MTSK. En cualquier caso, no conviene olvidar que esta decisión está mediatizada por las propias concepciones acerca de la matemática, de sus procesos de enseñanza y aprendizaje, de la visión de lo que es un profesor de matemáticas y de la propia identidad profesional, como ya hemos señalado.

Como punto de partida, podríamos considerar dos grandes referentes internacionales acerca del conocimiento de los profesores: los Standards for the Preparation of Middle Level Mathematics Teachers, del NCTM y el estudio TEDS-M, del IEA ${ }^{2}$ (International Association for the Evaluation of Educational Achievement).

El National Council of Teachers of Mathematics (NCTM, 2020), ha publicado recientemente los Standards for the Preparation of Middle Level Mathematics Teachers. Consiste en una revisión de los Estándares de la Asociación Profesional Especializada (SPA) para la preparación de maestros de Educación Primaria (1a a 5o), incorporando, a versiones anteriores, el estado de opinión y los resultados de la investigación en educación matemática en ese nivel educativo. Para ello, se hace eco de todas las aportaciones de la comunidad de educadores matemáticos, tanto en relación con los estándares curriculares y de evaluación, como los relativos a los procesos de enseñanza y aprendizaje de los contenidos matemáticos. Incorporan, así, propuestas de formación de profesores desde la perspectiva que solo una preparación adecuada coadyuvará en la tarea de involucrar a los estudiantes en formas de enseñanza y aprendizaje efectivas, asumiendo como imperativo que "que los nuevos profesores brinden a todos los estudiantes acceso a instrucción matemática de alta calidad que se enfoque en el significado, la comprensión y la aplicación del conocimiento procedimental (p. 4).

En esa línea proponen 4 estándares, enunciados en forma de conocimiento y acciones (proporcionando, de estas últimas, medios para evidenciarlas). El primer estándar se enuncia como

\footnotetext{
${ }^{2}$ IEA es una asociación independiente, compuesta por universidades, institutos o agencias ministeriales dedicadas a la investigación sobre evaluación educativa, que representan al sistema educativo de su país. El Instituto Nacional de Evaluación Educativa del Ministerio de Educación, Cultura y Deporte es miembro de la IEA.
} 
Conocer y comprender matemáticas significativas. Como acción observable de este conocimiento, establecen que los profesores han de demostrar y aplicar "su comprensión de los principales conceptos, procedimientos, conocimientos y aplicaciones de las matemáticas dentro y entre los dominios matemáticos de Números y Operaciones; Álgebra y funciones; Estadística y probabilidad; Geometría, trigonometría y medición”. Este planteamiento coincide con el concepto de comprensión profunda (Ma, 1999) de los conceptos y sus relaciones intra e inter-conceptuales, de los procedimientos matemáticos (incluyendo saber utilizarlos de forma "flexible, precisa, eficiente y apropiada", p. 6), de sus aplicaciones (incorporando la capacidad para formular y resolver problemas y una visión de la matemática como una herramienta útil en las actividades cotidianas) y el razonamiento lógico, unido a las capacidades de argumentar y justificar que tendrán que desarrollar en sus estudiantes. El estándar número 2 es, de alguna manera, un desarrollo de lo citado en relación con formular y resolver problemas, pues se enuncia como el "conocimiento y capacidad para aplicar los procesos matemáticos de resolución de problemas; razonar y comunicarse matemáticamente; y participar en modelos matemáticos” (p. 16), aplicando adecuadamente los recursos tecnológicos para ello.

Estos dos primeros estándares están centrados específicamente en el conocimiento del contenido matemático que ha de tener un profesor, el tercero lo hace en el ámbito del conocimiento didáctico del contenido. En efecto, bajo el enunciado conocer a los estudiantes y planificar el aprendizaje matemático, se manifiesta la intención de que los profesores integren el conocimiento matemático, antes descrito, con el correspondiente acerca del aprendizaje de los estudiantes para guiar la "planificar una instrucción matemática rigurosa y atractiva que respalde el acceso y el aprendizaje de los estudiantes ... [y que brinde] oportunidades equitativas y culturalmente receptivas para que todos los estudiantes aprendan y apliquen conceptos, habilidades y prácticas matemáticas” (p. 20). En este estándar, aparecen integrados el conocimiento del contenido con el conocimiento sobre su enseñanza y su aprendizaje, que se aprecia enfatizado si se analiza la justificación del estándar, que invita a atender a las potencialidades y limitaciones de los estudiantes a la hora de planificar la instrucción. Bajo la denominación de enseñar matemáticas significativas (cuarto y último estándar), se establece que los profesores marquen "rigurosos objetivos de aprendizaje de matemáticas, involucren a los estudiantes en un aprendizaje de alta demanda cognitiva, usen herramientas y representaciones específicas de matemáticas” (p. 30), a través prácticas de enseñanza efectivas y equitativas que conduzcan a un aprendizaje matemático potente. Con objeto de llegar a una inmensa mayoría de los estudiantes, esas prácticas han de propiciar una alta participación de los mismos, favoreciendo la comprensión conceptual y el uso fluido y flexible de los procedimientos.

En el primer estudio internacional comparativo acerca del conocimiento de carácter profesional de los futuros maestros y profesores de Secundaria, TEDS-M (Döhrmann et al., 2012; Tatoo et al., 2008), se pretendían mostrar comparativamente las características de los programas de formación del profesorado (Primaria y Secundaria), valorar su impacto en el conocimiento que realmente alcanzaban 
los profesores y extraer conclusiones acerca el futuro de la formación inicial. Su diseño se realizó con el apoyo de investigaciones previas acerca del conocimiento de los profesores, como MT21 Report (Mathematics Teaching for the 21st Century Project) (Schmidt et al., 2007) o el Learning Mathematics for Teaching Projects (Hill et al., 2004). Los informantes de este estudio se enfrentaron a cuestiones sobre las matemáticas y sobre sus procesos de enseñanza y aprendizaje, la mayoría de las cuales habían sido elaboradas expresamente para TEDS-M, aunque incorporaban otras de los estudios previos. Para las cuestiones de contenido matemático, la claridad y consistencia fue analizada y revisada por un equipo internacional de expertos a la luz del marco conceptual que soportaba el estudio (TIMSS 2007: Estudio Internacional de Tendencias en Matemáticas y Ciencias; el equipo de dirección del estudio, asesorado por otros investigadores, y sobre la base de la investigación en educación matemática, fue el responsable de la elaboración de las cuestiones sobre didáctica de la matemática.

Los tres dominios sobre los que se realizaron las cuestiones suponen un referente del contenido del conocimiento pretendido del profesor. En los dominios conceptuales del conocimiento matemático se incluyeron los bloques del contenido de la Educación Primaria y Secundaria como referentes (números y operaciones, geometría y medida, álgebra y funciones, azar y probabilidad) que, dado que no abarcaba todo el conocimiento matemático, se complementaba con los dominios o procesos cognitivos del conocimiento matemático (conocer, aplicar, razonar); por último, los dominios del conocimiento en didáctica de la matemática, incluían el conocimiento sobre el currículo de matemáticas (como la identificación de ideas clave y el establecimiento de objetivos e itinerarios), el de planificación de la enseñanza y el aprendizaje de las matemáticas (para predecir respuestas típicas de los estudiantes o seleccionar actividades apropiadas) y el conocimiento de la aplicación de la enseñanza de las matemáticas (en el diagnóstico de las respuestas del alumnado y de sus errores preconcebidos, el análisis y evaluación de las soluciones y argumentos matemáticos de los estudiantes o la explicación o representación de conceptos y procedimientos matemáticos).

Conocer y comprender matemáticas significativas junto con el conocimiento y capacidad para aplicar los procesos matemáticos, de los estándares del NCTM, mantienen una relación muy estrecha con los dominios conceptuales del conocimiento matemático y dominios o procesos cognitivos del conocimiento matemático, de TEDS-M; de la misma forma, conocer a los estudiantes y planificar el aprendizaje matemático junto con enseñar matemáticas significativas, la mantienen con los dominios del conocimiento en didáctica de la matemática, de TEDS-M.

Como puede verse, si se comparan estos dominios con los descritos más arriba en el modelo analítico MTSK, todos ellos forman parte de nuestro modelo, lo que nos da pie a establecer como un elemento clave del conocimiento del formador su conocimiento sobre MTSK y sobre las formas de ayudar a los futuros profesores a que lo construyan. Sin embargo, conviene resaltar que también se incluyen el conocimiento sobre los resultados de la investigación en educación matemática y el conocimiento sobre las creencias acerca de la matemática y de sus procesos de enseñanza y aprendizaje y 
su influencia a la hora de desarrollar en los futuros profesores la capacidad para formular y resolver problemas, junto con la visión de la matemática como una herramienta útil en las actividades cotidianas. Intentaremos abordar en lo que sigue algunos de estos aspectos desde una perspectiva no disjunta.

\section{HACIA UNA CARACTERIZACIÓN DEL CONOCIMIENTO DEL FORMADOR}

Las reflexiones sobre las características de un modelo de conocimiento del formador de profesores de matemáticas han nacido, como ya hemos señalado, muy influenciadas por los modelos subyacentes de conocimiento del profesor de matemáticas (Castro-Superfine et al., 2020; Chick \& Beswick, 2018; Leikin et al., 2018). Desde esa perspectiva es posible intentar ver un modelo para MTEK como una extensión del modelo para el conocimiento del profesor, algo así como que el MTEK contuviera ese conocimiento. Las aportaciones con base empírica de que disponemos en estos momentos (Chick \& Beswick, 2018), sin embargo, nos hacen pensar más bien en modelos con elementos comunes, por un lado, y diferenciados, por otro.

Así, hay aspectos del conocimiento del profesor que los formadores podrían no necesitar conocer, o tener de ellos un conocimiento diferente. Por ejemplo, podría decirse que el conocimiento de los formadores acerca de los aspectos curriculares de las matemáticas escolares, no tendría que coincidir con el correspondiente de los profesores, ya que su trabajo no los tiene como referencia inmediata. En cambio, los formadores necesitan tener un conocimiento detallado de los resultados de la investigación acerca de lo que es factible abordar en cada nivel educativo y de las limitaciones para ello (Beswick \& Chapman, 2012), lo que les proporciona criterios para discutir de forma crítica la estructura, contenidos y organización de un plan de estudios. Es decir, los formadores necesitan conocer el currículo escolar en relación con el conocimiento que quieren que adquieran los futuros profesores, pero no de la misma manera que un profesor de matemáticas necesita conocer el currículo. Otra componente adicional del conocimiento del formador identificado por Zazkis y Zazkis (2011) es la capacidad de empatizar con las dificultades con las matemáticas que manifiestan muchos de los profesores de primaria en formación.

Así que, como señalan Beswick y Chapman (2012), en general, parece que es más probable que el conocimiento de los formadores se superponga con el conocimiento de los profesores de matemáticas en lugar de contenerlo por completo.

En lo que sigue aportaremos algunas reflexiones acerca de posibles subdominios del conocimiento y sobre elementos comunes y diferenciales de MTEK y MTSK. No pretende ser un abordaje exhaustivo, sino más bien acerca de aquellos dominios cuya existencia en el conocimiento del formador podría tener un mayor grado de consenso; así, nos referiremos al dominio del conocimiento matemático, al dominio del conocimiento didáctico del contenido (de la formación) y, dentro de este, discutiremos algunos aspectos relativos al conocimiento del formador acerca de los futuros profesores y del proceso de enseñar a enseñar matemáticas. 


\section{EL CONOCIMIENTO MATEMÁTICO DEL FORMADOR}

Una de las finalidades de la educación matemática, que no la única, es la construcción del conocimiento matemático de los estudiantes. Cuando hablamos, en este punto, de conocimiento matemático, nos estamos refiriendo fundamentalmente al conocimiento de los temas del currículo, pero también a aquellos elementos de la estructura matemática y de la práctica matemática que resultan indispensables para la construcción de esos temas; así, por ejemplo, es necesario conectar las operaciones matemáticas entre ellas para que adquieran un significado, y también será necesario comprender el sentido de definir en matemáticas. Si nos detenemos un poco más en el conocimiento de los temas, debemos asumir que este implica el conocimiento de los procedimientos, de las definiciones, de las propiedades y algunos de sus fundamentos, el conocimiento de algunos fenómenos y aplicaciones y de los diferentes registros de representación que pueden utilizarse. Con todo, podemos pensar que es el conocimiento de los temas, en su conjunto, el que ocupa un mayor espacio en la Educación Matemática. Es cierto que el peso que los diferentes subdominios del conocimiento matemático tienen en la Educación Matemática, de los distintos niveles educativos, va variando. Cuando pasamos de Primaria a Secundaria y Bachillerato no solo incrementamos el peso del conocimiento de los temas (no solo en cantidad), también lo hacemos en el conocimiento de la estructura matemática y en el conocimiento de la práctica matemática. Podríamos decir, en esta primera aproximación, que el conocimiento matemático de cada etapa educativa contiene al conocimiento matemático de las etapas anteriores.

Hemos comentado muchas veces que si la educación matemática de la enseñanza obligatoria no se centrara en los aspectos procedimentales y abarcara de una forma más holística el conjunto del conocimiento de los temas, como hemos descrito más arriba, junto con las componentes estructural y sintáctica del conocimiento matemático, probablemente el conocimiento especializado a construir en los profesores que han de enseñar matemáticas estaría fundamentalmente centrado en el conocimiento didáctico del contenido matemático. Cabe detenerse aquí un poco en dos aspectos. En primer lugar, para tomar conciencia (como hemos anunciado previamente) de que el carácter generalista que la formación de profesores de Educación Primaria tiene (en muchos países, como es el caso de España) nos hace pensar en un conocimiento matemático mucho más integrado con el resto de los conocimientos disciplinares; citemos, por ejemplo, la importancia de integrar matemáticas y lenguaje para el proceso de comprensión de los problemas matemáticos. Esto nos hace ver una de las diferencias entre el conocimiento matemático de unos profesores y otros; la otra gran diferencia está, naturalmente, en la amplitud y profundidad del conocimiento, amplitud y profundidad que se percibe de forma natural en el conocimiento de la estructura matemática (por ejemplo, mayores conexiones de complejización y simplificación) y de la práctica matemática (conocimiento sobre las características de la demostración y sus tipos, por ejemplo). Aun así, la idea de inclusión antes expuesta para la construcción del conocimiento en los estudiantes sigue teniendo sentido. Añadamos que, estas diferencias en el conocimiento del dominio matemático de los 
profesores implican también diferencias en el dominio del conocimiento didáctico del contenido. Nuestros estudios empíricos con MTSK apuntan en esa dirección.

En nuestra reflexión sobre el conocimiento del formador de profesores de matemáticas podríamos tener en cuenta esta evolución del conocimiento matemático (de complejidad, profundidad y extensión crecientes) junto con el carácter emergente de nuevos conocimientos cuando cambiamos el sujeto de aprendizaje (estudiantes de Primaria, estudiantes de Secundaria y Bachillerato, futuros profesores). Sobre esto volveremos más adelante.

\section{EL CONOCIMIENTO DIDÁCTICO DEL CONTENIDO DEL FORMADOR}

Para entender el P”C”K del formador cabe reflexionar sobre la evolución de las acciones, que nos ayudarán a ver, en cada caso, como se integran y relacionan conocimientos de diferentes dominios. Las acciones de los profesores de matemáticas están orientadas a la construcción de conocimiento matemático. Para ello ponen en juego su conocimiento didáctico del contenido matemático para conseguir transformar la disciplina en formas útiles y adecuadas para sus estudiantes, generando tareas en las que los estudiantes interaccionen con el conocimiento matemático. Las acciones de los formadores, en cambio, están orientadas a la construcción de conocimiento especializado, generando tareas en las que el futuro profesor interacciona con situaciones de enseñanza y aprendizaje del contenido matemático. Así, mientras el profesor maneja el contenido matemático con la mirada puesta en el estudiante, el formador maneja simultáneamente el contenido matemático y el contenido didácticomatemático con dos miradas, una más directa puesta en el estudiante que forma (futuro profesor) y otra indirecta puesta en el estudiante a quien enseñará ese futuro profesor.

Olanoff (2011) nos ofrece un ejemplo de esta transferencia doble. Supongamos que el contenido en cuestión es la introducción a la multiplicación de fracciones. Lo que se pretende es que el estudiante de primaria aprenda, con significado, a multiplicar fracciones. Pero, para que los profesores sepan hacerlo, según Olanoff, el formador de profesores debe tener en cuenta el hecho de que los futuros profesores tendrán ideas preconcebidas sobre el tema (tanto desde la perspectiva del conocimiento matemático como de su enseñanza, como se detallará en el siguiente epígrafe), que su conocimiento será de carácter procedimental (y que, por tanto, trasmitirán ese carácter procedimental en su enseñanza) y que en algunos casos será erróneo. El formador de profesores debe ser capaz de construir conocimiento adecuado sobre los presupuestos anteriores, ayudar a aclarar los conceptos erróneos, así como introducir nuevas formas de ver la cuestión a través de modelos de representación adecuados. Tener un conocimiento amplio de las diferentes formas de modelizar o representar una multiplicación de fracciones permite a los formadores elegir ejemplos que ayudan a resaltar esas diferentes representaciones que los futuros profesores necesitan conocer para su trabajo en el futuro. Los formadores de profesores deben, además, ser capaces de hacer conexiones entre las relaciones entre las operaciones de números enteros y fracciones y ayudar a dotar de significado a los frágiles conocimientos algorítmicos que tienen 
muchos estudiantes para profesor. Los formadores de profesores deben ser capaces de extraer las características más importantes de un tema y hacer conexiones con el fin de ayudar a los futuros profesores a profundizar en su comprensión. Así, si ahora el tema fuera la división de fracciones, los formadores han de ser conscientes de la dificultad que entraña la división partitiva con fracciones, y esto a su vez podría ser utilizado para conectar más fácilmente con el algoritmo de invertir y multiplicar para la división fracciones.

Esto nos hace reflexionar sobre la emergencia de nuevos conocimientos que podríamos denominar conocimientos didácticos cuyo contenido es MTSK. Zaslavsky y Leikin (2004) discuten sobre una adaptación de la triada de enseñanza de Jaworski (1992, 1994) a la formación de profesores (es esta autora la que acuña el término MTEK). La creación de oportunidades para que los estudiantes aprendan matemáticas se transformaría en la creación de oportunidades para que los futuros profesores aprendan a enseñar matemáticas. Así, los elementos de la triada serían la gestión del aprendizaje de los profesores de matemáticas, contenidos desafiantes (no solo matemáticos) para ellos y sensibilidad hacia los mismos. Dentro de los contenidos desafiantes se encuentra la triada referida a los estudiantes, es decir, contenidos matemáticos desafiantes para los estudiantes, gestión del aprendizaje de los estudiantes y sensibilidad hacia los estudiantes. Las investigaciones que promueven el uso de casos o viñetas, elaboradas ad boc o extraídas de la realidad, en las que situar al futuro profesor ante problemas de enseñanza y aprendizaje de un contenido matemático se encontrarían en esta línea, poniendo de relieve estrategias que formarían parte de ese conocimiento para la enseñanza.

Asumimos así, de alguna manera, que el conocimiento del formador de profesores que enseñarán matemáticas tiene una componente matemática que, evolutivamente, ha ido ganando en profundidad y extensión (conceptos que hemos intentado desgranar anteriormente), un conocimiento ha de estar ligado al conocimiento didáctico del contenido matemático que pretende construir en sus futuros profesores (lo que puede significar un conocimiento profundo de las relaciones entre los diferentes subdominios de MTSK) y, junto a ello, encontramos una componente de un conocimiento didáctico que le permita construir MTSK en los futuros profesores. Es este último, por tanto, el que requiere de un mayor nivel de reflexión por nuestra parte.

Para Abell et al. (2009) existe una réplica de PCK para los formadores de profesores de matemáticas. En este caso, el conocimiento de la materia que necesita un formador incluye tanto el contenido matemático como el conocimiento para su enseñanza. Se incluyen en este $\mathrm{P}$ "C $\mathrm{K}^{3}$ diferentes orientaciones hacia el aprendizaje de la matemática, el conocimiento de cómo aprenden los futuros profesores, el conocimiento de los planes de estudio para la formación de profesores, el conocimiento de las estrategias de instrucción para la enseñanza de los futuros profesores, y el conocimiento de la evaluación de los futuros profesores.

${ }^{3}$ Como ya hemos señalado, Chick y Beswick (2018) lo denominan MTEPCK, para diferenciarlo del PCK de los profesores. 
Appova y Taylor (2019) enumeran un elenco de acciones bajo las que podemos analizar elementos del P"C”K del formador. El formador ha de enseñar a usar recursos manipulativos como herramientas de instrucción, a usar diferentes modelos de representación, ayudar a comprender un vocabulario amigable para los estudiantes como táctica desarrollo del lenguaje matemático, ayudar a prender sobre modelos de enseñanza ejemplares extraídas de sesiones reales de enseñanza, ayudar a entender las concepciones, las potencialidades, los conceptos erróneos y los errores ingenios de los estudiantes, ayudar a aprender acerca de las teorías de la cognición y el desarrollo de los estudiantes y a desgranar los elementos del currículo del nivel en el que van a enseñar, con una comprensión profunda del contenido que les permita hacer conexiones matemáticas más allá de lo recogido en el plan de estudios. Desde nuestra perspectiva, esto implica un conocimiento de cómo enseñar el PCK del profesor; pero entendemos que el P”C”K del formador va más allá.

\section{EL CONOCIMIENTO SOBRE EL CONOCIMIENTO Y LAS CONCEPCIONES DE LOS FUTUROS PROFESORES}

Pensemos en un ejemplo. Cuando en MTSK hablamos de las características del aprendizaje de las matemáticas (KFLM), consideramos (entre otros aspectos) el conocimiento del profesor sobre las potencialidades y dificultades de aprendizaje de sus estudiantes y, en esas dificultades, incluimos los posibles conceptos erróneos que pueden tener, como podría ser la idea de que, en la comparación de dos figuras planas, a mayor perímetro, siempre habrá mayor área (o viceversa). Si intentamos pensar en un subdominio equivalente para el MTEK, la categoría anteriormente descrita también podría seguir teniendo sentido (son muchos los estudios que ponen de relieve que los estudiantes para profesor arrastran errores propios de la educación obligatoria); sin embargo, tendríamos que incluir también aquí el conocimiento acerca de las concepciones de los futuros profesores ( $y$ ahora no me refiero a conceptos erróneos o conocimiento erróneo, sino a concepciones en sentido estricto) acerca de los procesos de enseñanza y aprendizaje. Por ejemplo, como formadores, somos conscientes de la sobrevaloración de los futuros profesores hacia los recursos didácticos, en el sentido de que su mera manipulación conduce a aprendizaje (tendencia espontaneísta de muchos candidatos a maestro de Primaria), o la creencia acerca de la suficiencia del conocimiento matemático sólido, para la enseñanza (tendencia tradicionaltecnológica) en los candidatos a profesor de Secundaria. Esto forma parte del elenco de conocimientos de un formador y no tiene por qué formar parte del conocimiento de un profesor.

De alguna manera, este ejemplo nos muestra cómo en el conocimiento del formador estamos uniendo elementos de dos dominios (PCK y creencias) del conocimiento del profesor, pero esa fusión adquiere un cuerpo propio dentro del conocimiento del formador acerca de las "características del conocimiento del futuro profesor", que es lo que nos da pie a pensar en una denominación como la que da título a esta reflexión. 
Appova y Taylor (2019) apuntan algo similar cuando, dentro del PCK del formador, incluyen en una misma categoría: enseñar a usar recursos manipulativos como herramientas de instrucción, enseñar a usar diferentes modelos de representación (hibridando KMT y KoT, en lo que podría llamarse conocimiento sobre la enseñanza de MTSK, que daría pie a reflexionar sobre otro subdominio, diferente de MTEK, del que centra este apunte).

Es posible (y es algo que hay que explorar) que los futuros subdominios del MTEK contengan híbridos de categorías diferentes de subdominios de MTSK y pensamos que esto debe animarnos a tener la mente abierta a esas posibilidades.

\section{EL CONOCIMIENTO DE ENSEÑAR A ENSEÑAR MATEMÁTICAS}

El formador tiene como cometido enseñar a enseñar matemáticas (que desde la perspectiva de MTSK está vinculado a enseñar conocimiento de la enseñanza de las matemáticas). Es en este aspecto donde la experiencia del formador como docente (del nivel formativo del que ahora forma profesores) se muestra como esencial. Tzur (2001, citado en Zopf, 2010) describe la evolución del conocimiento del formador, desde aprendiz de matemáticas a profesor de matemáticas y, desde ahí, a formador de profesores de matemáticas y posteriormente a mentor de educadores de profesores de matemáticas. Esta experiencia del formador puede permitirle un nivel de comprensión del conocimiento matemático desde una perspectiva mucho más amplia. Zazkis (2011) propone la idea de reevaluar conceptos familiares, reconsiderando ideas sostenidas anteriormente y, en última instancia, reconstruyendo el conocimiento de los futuros profesores. Argumenta para ello que este reaprendizaje también implica nuevas formas de aprender el contenido y mejorar la comprensión del contenido aprendido previamente desde perspectivas exclusivas para la enseñanza. En este contexto, enseñar a enseñar matemáticas podría verse como un isomorfismo didáctico mediante el que el formador aborda el reaprendizaje de sus futuros profesores ayudándoles a reflexionar no solo sobre el contenido que aprenden sino más bien sobre el proceso que utilizan para ello, de forma que puedan luego transferir ese proceso en el trabajo con sus estudiantes.

Castro-Superfine et al. (2020) nos muestran tres ejemplos que ilustran esta idea. En el primer ejemplo destacan una tarea utilizada para ayudar a los futuros profesores a volver a aprender un contenido matemático haciéndolos participar en el pensamiento poco convencional de un estudiante. En el segundo ejemplo describen cómo los formadores diseñaron e implementaron una tarea que facilitó el reaprendizaje de las fracciones por parte de los futuros profesores cuando les animaron a desarrollar estrategias con sentido en vez de procedimiento rutinarios. Y, por último, el tercer ejemplo se centra en la elaboración y discusión de tareas para los futuros profesores para ayudarles a desarrollar una visión del contenido como objeto de enseñanza y aprendizaje. Aunque estos ejemplos pueden no resultar especialmente novedosos, juntos ilustran que el trabajo de los formadores implica considerar cuidadosamente las tareas matemáticas y las prácticas pedagógicas que apoyan el aprendizaje y reaprendizaje de los futuros profesores. Este trabajo de los formadores requiere conocimiento 
especializado que tiene en cuenta la reconstrucción del conocimiento de los estudiantes adultos que, aunque incluye el conocimiento para enseñar matemáticas, es sustancialmente diferente. No solo son diferentes los propósitos de diseñar tareas para futuros profesores de los propósitos de diseñar tareas para estudiantes, lo que ya en sí muestra las diferencias entre el conocimiento necesario para enseñar matemáticas a los estudiantes (KMT) y el conocimiento necesario para enseñar conocimiento especializado a los futuros profesores; es ese isomorfismo que nos muestra el conocimiento de los formadores para apoyar el reaprendizaje de matemáticas de los futuros profesores cuyo propósito es mostrarles cómo apoyar el aprendizaje de las matemáticas por parte de sus futuros estudiantes.

\section{VÍAS PARA ACCEDER AL CONOCIMIENTO DEL FORMADOR}

Hasta ahora, nuestras aproximaciones al conocimiento de los formadores de profesores que enseñarán matemáticas se ha orientado desde cuatro perspectivas: desde la propia reflexión teórica (Escudero-Ávila et al., 2021), con la sensibilidad que nos da nuestra experiencia en el estudio de MTSK, desde las orientaciones curriculares, nacionales, internacionales o estrictamente académicas acerca de la estructura y el contenido de la formación que han de realizar, desde el propio contenido de la formación que observamos en los formadores expertos (procurando extraer aquellos conocimientos que no comparte con el profesor) (Pascual et al., 2019), o desde las opiniones manifestadas por los diferentes agentes (vinculados al conocimiento de educación matemática) que intervienen en la formación de profesores (Montes \& Contreras, 2019).

Un elemento implícito que se ha surgido en algunos de los trabajos, realizados desde esas perspectivas, es profundizar en el conocimiento que sustentan las acciones que los formadores realizan o estimamos susceptibles de realizar en la formación, desde nuestra comprensión del conocimiento, como conocimiento en la acción.

Profundizar en el conocimiento desde el análisis de las acciones nos fue útil en el algunas de las fases de consolidación de MTSK; por ello, pensamos que, si tomamos como punto de partida acciones específicas de los formadores, su análisis nos puede proporcionar información acerca del conocimiento que encierran. Cada una de estas acciones irán encaminadas a construir MTSK en los futuros profesores. Necesitamos aislar, del análisis del conocimiento, ese MTSK y encontrar ese otro conocimiento específico que lo sustenta.

Enumeremos estas acciones que deberían orientarnos en nuestro acercamiento a los posibles conocimientos que las sustentan:

a) Reflexión sobre los elementos de enseñanza y aprendizaje inmersos en los procesos de enunciar y resolver problemas.

b) Reflexión sobre los elementos de enseñanza y aprendizaje inmersos en los procesos de selección y uso de recursos. 
c) Análisis de las implicaciones de los elementos teóricos de la educación matemática (Niveles de Van Hiele, tipología de problemas aritméticos, estrategias personales de adición y sustracción...) para el diseño de actividades del aula de Primaria.

d) Problematización de la práctica que posibilite la reflexión, mediante la selección, diseño y secuenciación de tareas matemáticamente relevantes para el aprendizaje de los futuros profesores, dotando de significado a todos los conceptos e ideas relacionadas con los procesos de E-A involucrados (K”MTSK”T) a la vez que proporciona al profesorado en formación ejemplos y contextos de uso de las matemáticas que sean útiles para su alumnado.

e) Problematización de la práctica para plantear o seleccionar situaciones ejemplares (dentro del dominio del conocimiento pedagógico del contenido de las matemáticas escolares), que se supone ha de generar dudas en los futuros profesores (KFL”MTSK”).

f) Desarrollo de competencias profesionales como Noticing (que puede ayudar a la problematización de la práctica o uso de narrativas.

g) La propia investigación sobre la práctica (no como desarrollo de herramientas formativas, sino como acción académica del formador), es una acción propia del formador. Por tanto, los conocimientos que encierra han de ser objeto de estudio.

También parece necesario profundizar en el análisis de las fuentes del conocimiento del formador. Una de las fuentes que, de forma implícita, se ha puesto de relieve como potente es la propia práctica como profesor en ese nivel en el que ahora se actúa como formador, lo que hace que consideremos la formación del formador como parte del desarrollo profesional de un profesor. Otra fuente de conocimiento proviene de la investigación en educación matemática. Asimismo, el análisis intersubjetivo de las acciones de otros profesores y de las propias acciones de profesores en formación constituye una fuente de información acerca de la identidad profesional; de hecho, el conocimiento sobre las identidades profesionales (susceptibles de ser desarrolladas) y sobre la propia identidad profesional puede ya considerarse como componente del conocimiento del formador.

Se hace necesaria abundante investigación empírica que, teniendo como informantes a diferentes formadores, arroje más luz acerca de este problema al que, en este artículo solo hemos hecho una modesta aproximación teórica. Sí parece razonable que esos informantes compartan como principio básico que forman un profesional que habrá de considerar la matemática como objeto de enseñanza y aprendizaje.

\section{ACLARATORIA}

El autor no tiene conflicto de interés que declarar. El artículo ha sido financiado con recursos propios del autor. 


\section{REFERENCIAS}

Abell, S. K., Rogers, M. A. P., Hanuscin, D. L., Lee, M. H., \& Gagnon, M. J. (2009). Preparing the next generation of science teacher educators: A model for developing PCK for teaching science teachers. Journal of Science Teacher Education, 20(1), 77-93. https://doi.org/10.1007/s10972-008-9115-6

Appova, A., \& Taylor, C.E. (2019). Expert mathematics teacher educators' purposes and practices for providing prospective teachers with opportunities to develop pedagogical content knowledge in content courses. Journal of Mathematics Teacher Education, 22, 179-204. https://doi.org/10.1007/s10857-017-9385-z

Ball, D. L., \& Bass, H. (2003). Toward a Practice-Based Theory of Mathematical Knowledge for Teaching. En Proceedings of Annual Meeting of Canadian Mathematics Education Study Group (pp. 3-14). CMESG.

Ball, D. L., \& Bass, H. (2009). With an eye on the mathematical horizon: knowing mathematics for

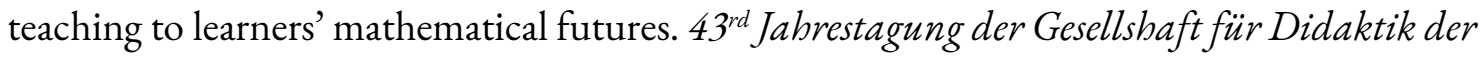
Mathematik. Oldenburg, Germany

Ball, D. L., Thames, M. H., \& Phelps, G. (2008). Content knowledge for teaching: What makes it special? Journal of Teacher Education, 59(5), 389-407. https://doi.org/10.1177/0022487108324554

Bass, H. (1997). Mathematician as educators. Notices of the AMS, 44(1), 18-21.

Beswick, K., \& Chapman, O. (2015). Discussion group 12: Mathematics teacher educators' knowledge for teaching. Conducted at the 12th International Congress on Mathematics Education held in Seoul, South Korea. (pp. 29-32) https://doi.org/10.1007/978-3-319-12688-3

Carrillo, J., Climent, N., Montes, M., Contreras, L. C., Flores-Medrano, E., Escudero-Ávila, D., VascoMora, D., Rojas, N., Flores, P., Aguilar-González, A. Ribeiro, M., \& Muñoz-Catalan, M. C. (2018). The Mathematics Teacher's Specialised Knowledge (MTSK) model. Research in Mathematics Education, 20(3), 236-253. https://doi.org/10.1080/14794802.2018.1479981

Carrillo, J., Contreras, L. C., \& Flores, P. (2013). Un modelo de conocimiento especializado del profesor de matemáticas. En L. Rico, M. C. Cañadas, J. Gutiérrez, M. Molina, \& I. Segovia (Eds.), Investigación en Didáctica de la Matemática. Libro homenaje a Encarnación Castro (pp. 193200). Comares.

Castro-Superfine, A., Prasad, P. V., Welder, R. M., Olanoff, D., \& Eubanks-Turner, C. (June 2020). Exploring mathematical knowledge for teaching teachers: Supporting prospective teachers' relearning of mathematics. En A. Appova, R. M. Welder, and Z. Feldman, (Eds.), Supporting 
Mathematics Teacher Educators' Knowledge and Practices for Teaching Content to Prospective (Grades K-8) Teachers. Special Issue: The Mathematics Enthusiast, ISSN 1551-3440, vol. 17, nos. 2 \& 3, pp. 367-402. Scholar Works: University of Montana. Retrieve (open access) https://scholarworks.umt.edu/tme/vol17/iss2/3/

Chick, H., \& Beswick, K. (2013). Educating Boris: An examination of pedagogical content knowledge for mathematics teacher educators. En V. Steinle, L. Ball, \& C. Bardini (Eds.), Mathematics education: Yesterday, today and tomorrow (Proceedings of the 36th annual conference of the Mathematics Education Research Group of Australasia, eBook, pp. 170-177). MERGA, Inc.

Chick, H., \& Beswick, K. (2018). Teaching teachers to teach Boris: a framework for mathematics teacher educator pedagogical content knowledge. Journal of Mathematics Teacher Educator, 21, 475-499. https://doi.org/10.1007/s10857-016-9362-y

Döhrmann, M., Kaiser, G., \& Blömeke, S. (2012). The conceptualization of mathematics competencies in the international teacher education study TEDS-M. ZDM Mathematics Education, 44, 325-340. https://doi.org/10.1007/s11858-012-0432-z

Escudero-Ávila, D., Montes, M., \& Contreras, L. C. (2021). What do Mathematics Teacher Educators need to know? Reflections emerging from the content of mathematics teacher education. En M. Goos, \& K. Beswick (Eds.), The learning and development of mathematics teacher educators: international perspectives and challenges. Springer. https://:doi.org/10.10007/978-3-030-62408-8 2

Godino, J. D. (2009). Categorías de análisis de los conocimientos del profesor de matemática. UNION, Revista Iberoamericana de Educación Matemática, 20, 13-31 https://union.fespm.es/index.php/UNION/issue/view/27/25

Hill, H., Schilling, S., \& Ball, D. (2004). Developing measures of teachers' mathematical knowledge for teaching. Elementary School Journal, 105(1), 11-30. https://doi.org/10.1086/428763

INECSE (2005). PISA 2003. Pruebas de Matemáticas y de Solución de Problemas. INECSE.

Jaworski, B. (1992). Mathematics teaching: What is it? For the Learning of Mathematics, 12(1), 8-14.

Jaworski, B. (1994). Investigating mathematics teaching: A constructivist enquiry. The Falmer Press.

Jaworski, B. (2008). Development of the mathematics teacher educator and its relation to teaching development. En B. Jaworkki, \& T. Wood (Eds.), The mathematics teacher educator as a developing professional (p. 335-361; Volume 4, The international handbook of mathematics teacher education). Sense Publishers. 
Leikin, R., Zazkis, R., \& Meller, M. (2018). Research mathematicians as teacher educators: focusing on mathematics for secondary mathematics teachers. Journal of Mathematics Teacher Educator 21, 451-473. https://doi.org/10.1007/s10857-017-9388-9

Ma, L. (1999). Knowing and teaching elementary mathematics: Teachers' understanding of fundamental mathematics in China and the United States. Lawrence Erlbaum Associates. https://doi.org/10.4324/9781410602589

Montes M., \& Contreras L. C. (2019). Las creencias de los formadores de profesores que enseñarán matemáticas sobre el contenido y la estructura de la formación inicial de los profesores de secundaria. En J. Carrillo, M. Codes, \& L. C. Contreras (Eds.), IV Congreso Iberoamericano sobre Conocimiento Especializado del Profesor de Matemáticas (pp. 14-23). Universidad de Huelva Publicaciones.

Olanoff, D. (2011). Mathematical Knowledge for Teaching Teachers: The Case of Multiplication and Division of Fractions. Mathematics Disertations. Paper 64.

Pascual, M. I., Montes, M., \& Contreras, L. C. (2019). Un acercamiento al conocimiento del formador de profesores de matemáticas. In J. M. Marbán, M. Arce, A. Maroto, J. M. Muñoz-Escolano and Á. Alsina (Eds.), Investigación en Educación Matemática XIX (pp. 473-482). SEIEM.

Schwab, J. J. (1978). Science, curriculum and liberal education. University of Chicago Press.

Shulman, L. S. (1986). Those who understand: knowledge growth in teaching. American Educational Research Association, 15(2), 4-14. https://doi.org/10.3102/0013189X015002004

Shulman, L. S. (2005). Conocimiento y enseñanza: fundamento de la nueva reforma. Profesorado. Revista de Currículum y Formación de Profesorado, 9(2), 1-30.

http://www.ugr.es/ recfpro/rev92ART1.pdf

Schmidt, W., Tatto, M. T., Bankov, K., Blomeke, S., Cedillo, T., Cogan, L., Han, S. I., Houang, R., Hsieh, F. J., Paine, L., Santillan, M., \& Schwille, J. (2007, December). The preparation gap: Teacher education for middle school mathematics in six countries (MT21 report). East Lansing, MI: Michigan State University (NSF REC 0231886/January 2003).

http://usteds.msu.edu/MT21Report.pdf

Tatto, M. T., Schwille, J., Senk, S., Ingvarson, L., Peck, R., \& Rowley, G. (2008). Teacher Education and Development Study in Mathematics (TEDS-M): Policy, practice, and readiness to teach primary and secondary mathematics. Conceptual framework. Teacher Education and Development International Study Center, College of Education, Michigan State University. https://www.iea.nl/sites/default/files/2019-04/TEDS-M_Framework.pdf 
The National Council of Teachers of Mathematics (2020). Standards for the Preparation of Middle Level Mathematics Teachers. NCTM.

https://www.nctm.org/uploadedFiles/Standards and Positions/NCTM Middle School 2020 Final.pdf

Tzur, R. (2001). Becoming a mathematics teacher-educator: Conceptualizing the terrain through selfreflective analysis. Journal of Mathematics Teacher Education, 4, 259-283.

https://doi.org/10.1023/A:1013314009952

Zaslavsky, O., \& Leikin, R. (2004). Professional development of mathematics teacher educators: growth through practice. Journal of Mathematics Teacher Education 7, 5-32. https://doi.org/10.1023/B:JMTE.0000009971.13834.e1

Zazkis, R. (2011). Relearning mathematics: a challenge for prospective elementary school teachers. Information Age Publishing.

Zazkis, R., \& Zazkis, D. (2011). The significance of mathematical knowledge in teaching elementary methods courses: perspectives of mathematics teacher educators. Educational Studies in Mathematics, 76(3), 247-263. https://doi.org/10.1007/s10649-010-9268-z

Zopf, D. (2010). Mathematical knowledge for teaching teachers: the mathematical work of and knowledge entailed by teacher education. Unpublished doctoral dissertation.

http://deepblue.lib.umich.edu/bitstream/2027.42/77702/1/dzopf_1.pdf

\section{Cómo citar este artículo:}

Contreras, L. C. (2021). Una aproximación a un modelo de conocimiento del formador de profesores de matemáticas. Revista Venezolana de Investigación en Educación Matemática (REVIEM), 1(1), e202101. https://doi.org/10.54541/reviem.v1i1.12 
(c) (9) Copyright (C) 2021. Luis Carlos Contreras. Esta obra está protegida por una licencia Creative Commons 4.0. International (CC BY 4.0).

Usted es libre para Compartir — copiar y redistribuir el material en cualquier medio o formato - y Adaptar el documento - remezclar, transformar y crear a partir del material — para cualquier propósito, incluso para fines comerciales, siempre que cumpla la condición de:

Atribución: Usted debe dar crédito a la obra original de manera adecuada, proporcionar un enlace a la licencia, e indicar si se han realizado cambios. Puede hacerlo en cualquier forma razonable, pero no de forma tal que sugiera que tiene el apoyo del licenciante o lo recibe por el uso que hace de la obra.

$\underline{\text { Resumen de licencia - Texto completo de la licencia }}$ 